Notes

\title{
Fullerene as a Standard Sample for Adjusting the NMR Spectral Resolution in Multiple-Quantum Magic-Angle-Spinning Experiments
}

\author{
Yuichi Shimoikeda, ${ }^{*}$ Hiroaki Utsumi, ${ }^{*}$ Koji Saito, ${ }^{* *}$ Tadashi ShImizu, ${ }^{* * *}$ and Toshihito NakaI* \\ *JEOL Ltd., 3-1-2 Musashino, Akishima, Tokyo 195-8558, Japan \\ **Nippon Steel Corporation, Futtsu, Chiba 293-8511, Japan \\ ***National Institute for Material Science, 3-13 Sakura, Tsukuba, Ibaraki 305-0003, Japan
}

\begin{abstract}
A method using fullerene for adjusting the NMR spectral resolution for multiple quantum magic angle spinning (MQMAS) experiments is proposed. To observe its ${ }^{13} \mathrm{C}$ MAS signal, it is not necessary to apply ${ }^{1} \mathrm{H}$ decoupling, unavailable with single-resonance MQMAS probes. Although ${ }^{13} \mathrm{C} T_{1}$ of fullerene is rather long, a recycle time of $5 \mathrm{~s}$ in shimming yields its signal with sufficiently high sensitivity if setting the appropriate Ernst angles corresponding to magnetic fields. It is demonstrated that so-adjusted high resolution is reflected in the ${ }^{87} \mathrm{Rb}$ MQMAS spectra of $\mathrm{RbNO}_{3}$.
\end{abstract}

(Received March 26, 2007; Accepted June 14, 2007; Published August 10, 2007)

\section{Introduction}

Spectral resolution is one of the most essential factors in determining the quality of NMR spectra. It is almost selfevident that higher resolution of MQMAS (multiple quantum magic angle spinning) spectra ${ }^{1,2}$ can be obtained, when higher resolution of a standard sample is achieved. Therefore, it is practically important to find a sample that is appropriate for adjusting the spectral resolution.

To observe MQMAS spectra, the strongest possible RF field is required to enhance the multiple-quantum coherence and to avoid spectral distortion. ${ }^{2-5}$ For this reason, a special singleresonance probe, instead of a CPMAS double-resonance probe, is desirably utilized. Accordingly, we can not enjoy high-power ${ }^{1} \mathrm{H}$ decoupling with such a special probe. It follows that a sample containing ${ }^{1} \mathrm{H}$ nuclei, such as adamantane, is not suitable for adjusting the resolution without ${ }^{1} \mathrm{H}$ high-power decoupling. In other words, by using the novel sample without containing ${ }^{1} \mathrm{H}$ nuclei, one can apply the longest possible acquisition time without ${ }^{1} \mathrm{H}$ decoupling in prolonging the FID by shimming.

We summarize the criteria for such a standard sample with which the spectral resolution is adjusted, as follows:

(1) No ${ }^{1} \mathrm{H}$ nuclei should be contained.

(2) No large quadrupolar interaction should be involved; spin$1 / 2$ nuclei or highly symmetric quadrupolar nuclei should be contained.

(3) The intrinsic MAS resonance line should be sufficiently sharp.

(4) The recycle time could be as short as 5 to $10 \mathrm{~s}$ and should yield a sufficiently high sensitivity signal with a single transient.

(5) The sample should be a stable solid at room temperature and pressure.

(6) The sample should be commercially available at a reasonable cost.

$\dagger$ To whom correspondence should be addressed.

E-mail: tnakai@jeol.co.jp
In this article, we show that fullerene (C60) is an appropriate sample for adjusting the spectral resolution; fullerene fulfills all of the above criteria.

\section{Experimental}

In the NMR experiments, we used the JEOL ECA/ECX series of NMR spectrometers; the ${ }^{1} \mathrm{H}$ resonance frequencies were 400 , 500, 600, 700, and $930 \mathrm{MHz}$, and the corresponding magnetic field strengths were $9.4,11.7,14.1,16.4$, and $21.9 \mathrm{~T}$. In the case of $14.1 \mathrm{~T}$, the ${ }^{13} \mathrm{C}$ and ${ }^{87} \mathrm{Rb}$ resonance frequencies were 150.9 and $196.4 \mathrm{MHz}$, respectively. Also, a JEOL singleresonance MQMAS probe was used. All of the NMR experiments were conducted at room temperature and pressure.

We used fullerene to adjust the spectral resolution, or shimming. In the MQMAS experiments, we used $\mathrm{RbNO}_{3}$ as a test sample to check the MQMAS spectral resolution. Both fullerene and $\mathrm{RbNO}_{3}$ were purchased from Tokyo Kasei Kogyo and used without further purification.

\section{Results and Discussion}

Figure 1 shows the ${ }^{13} \mathrm{C}$ MAS spectra of fullerene at $14.1 \mathrm{~T}$, of which the resolution is adjusted in Fig. 1(a) and not adjusted in Fig. 1(b). The corresponding ${ }^{87} \mathrm{Rb}$ MQMAS spectra of $\mathrm{RbNO}_{3}$ are shown in Figs. 1(c) and (d), respectively. The linewidth of fullerene is directly reflected in the target MQMAS spectra, as obviously recognized in the $F_{1}$ isotropic projections. This shows that adjusting the spectral resolution prior to MQMAS experiments is necessary. The details of the adjustment are discussed and shown below.

The sharpness of the resonance line is required for the sample with which the resolution is adjusted. Table 1 shows the FWHH (full width at half hight) of ${ }^{13} \mathrm{C}$ MAS resonance lines of fullerene at various sample spinning frequencies at $14.1 \mathrm{~T}$, which we realized through elaborate shimming; the values of the FWHH indicate the highest possible resolution that we 

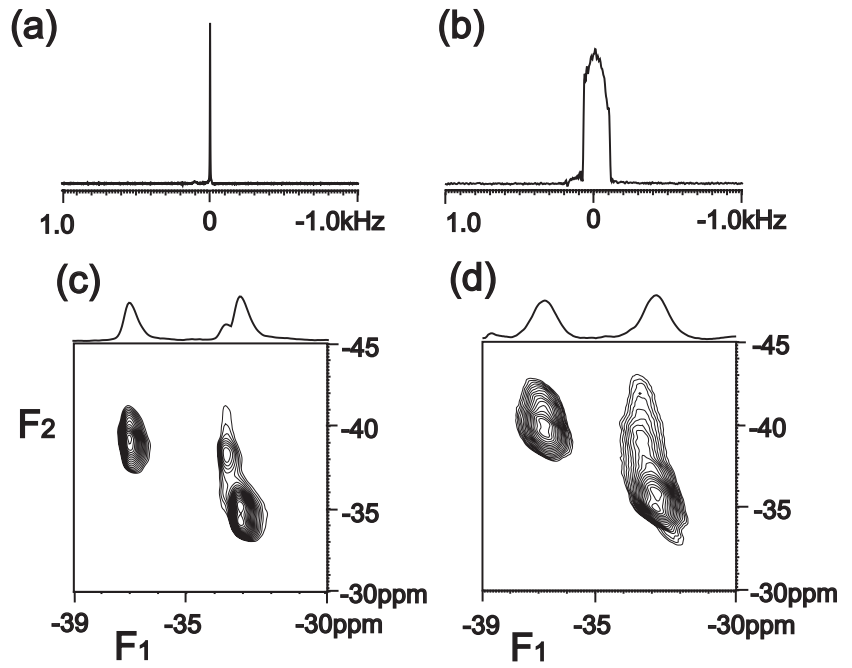

Fig. $1{ }^{13} \mathrm{C}$ MAS spectra of fullerene at a spinning frequency of 5 $\mathrm{kHz}$ with which the spectral resolution is adjusted up to FWHH of $3.3 \mathrm{~Hz}$ (a), and not adjusted and having FWHH of $170 \mathrm{~Hz}$. Under these ${ }^{13} \mathrm{C}$ resolution, or the shimming conditions, the ${ }^{87} \mathrm{Rb}$ MQMAS spectra of $\mathrm{RbNO}_{3}$ are observed at a spinning frequency of $19 \mathrm{kHz}$ and shown in (c) and (d), respectively. $F_{1}$ isotropic projections of the individual 2D spectra reflect the inhomogeneity of magnetic fields represented by ${ }^{13} \mathrm{C}$ resolution shown in Figs. 1(a) and (b).

achieved. Once the sample is spun, the resonance line becomes sharp from 200 to $2 \mathrm{~Hz}$. Although further line narrowing may be expected at faster spinning frequencies, $5 \mathrm{kHz}$ is experimentally found to be sufficient, as shown in Fig. 1 and Table 1.

As a sample with which the resolution is adjusted, one of the criteria is a comparatively short $T_{1}$ relaxation time for a fast recycle delay. Table 2 gives the ${ }^{13} \mathrm{C} T_{1}$ values of fullerene obtained at various magnetic fields $\left(B_{0}\right)$ from 9.4 to $21.9 \mathrm{~T}$. Obviously, a higher magnetic field makes the relaxation time $\left(T_{1}\right)$ shorter, in contrast to the ordinary rigid solid samples. From Table 2, we can judge that the signal of fullerene is more easily acquired at a higher magnetic field. It is reported ${ }^{6-9}$ that the $T_{1}$ relaxation of fullerene at room temperature is dominated by the chemical-shift anisotropy, and the value of $T_{1}$ is consequently proportional to $1 / B_{0}{ }^{2}$, which elucidates our observation.

The $T_{1}$ value of $25 \mathrm{~s}$ at a high field of $14.1 \mathrm{~T}$ is still long for the recycle delay of shimming. However, the combination of faster recycle delays and small flip angles can optimize the experimental condition. For example, if the recycle delay is fixed at $5 \mathrm{~s}$, the Ernst angles ${ }^{10}$ for the individual magnetic fields are evaluated as in Table 3 ; the sensitivity of the ${ }^{13} \mathrm{C}$ signal intensity is $31.6 \%$ compared with that of the almost completely recovered signal using a 90 degree pulse after $125 \mathrm{~s}, 5$-times that of $T_{1}$. This sensitivity is sufficiently high, and we can repeat the shimming with a recycle delay of $5 \mathrm{~s}$. In fact, the resolution is adjusted so as to obtain the spectrum shown in Fig. 1(a).

Also, the relative sensitivities of fullerene at the abovementioned various magnetic fields normalized with the signal intensity at $14.1 \mathrm{~T}$ are given in Table 3 . A higher magnetic field makes the signal sensitivity higher, partly because of a shorter relaxation time $\left(T_{1}\right)$. However, even at a low field of $9.4 \mathrm{~T}$, more than $50 \%$ of the sensitivity compared with that at $14.1 \mathrm{~T}$ is expected, which indicates that fullerene can still be used for shimming.

When MQMAS probes are not available, prevailing CPMAS double-resonance probes ordinarily equipped may instead be
Table 1 Linewidth (FWHH) of the ${ }^{13} \mathrm{C}$ MAS resonance line of fullerene at various sample spinning frequencies, $v_{\mathrm{R}}$ observed at $14.1 \mathrm{~T}$

\begin{tabular}{cc}
\hline$v_{\mathrm{R}} / \mathrm{kHz}$ & $\mathrm{FWHH} / \mathrm{Hz}$ \\
\hline 15 & 1.5 \\
10 & 1.9 \\
5 & 2.0 \\
0 & 200 \\
\hline
\end{tabular}

Table $2{ }^{13} \mathrm{C}$ longitudinal relaxation time $\left(T_{1}\right)$ of fullerene at various magnetic fields, $B_{0} \mathrm{~T}\left(v_{0} \mathrm{MHz}\right.$ for ${ }^{1} \mathrm{H}$ Larmor frequencies)

\begin{tabular}{cl}
\hline$B_{0} / \mathrm{T}\left(v_{0} / \mathrm{MHz}\right)$ & $T_{1} / \mathrm{s}$ \\
\hline $9.4(400)$ & 50 \\
$11.7(500)$ & 30 \\
$14.1(600)$ & 25 \\
$16.4(700)$ & 20 \\
$21.9(930)$ & 13.3 \\
\hline
\end{tabular}

Table 3 Ernst angles ( $\theta_{\mathrm{EA}}$ degree) in the case of a recycle time of $5 \mathrm{~s}$ at various magnetic fields, $B_{0} \mathrm{~T}\left(v_{0} \mathrm{MHz}\right.$ for ${ }^{1} \mathrm{H}$ Larmor frequencies), and the sensitivities relative to the full recovery after five times of $T_{1}$ at each field, $(S / N)_{\mathrm{EA}} /(S / N)_{\mathrm{FR}}$

\begin{tabular}{cccc}
\hline$B_{0} / \mathrm{T}\left(v_{0} / \mathrm{MHz}\right)$ & $\theta_{\mathrm{EA}} /$ degree & $\begin{array}{c}(S / N)_{\mathrm{EA}} / \\
(S / N)_{\mathrm{FR}}, \%\end{array}$ & $\begin{array}{c}(S / N)_{\mathrm{EA}} / \\
(S / N)_{\mathrm{EA} @ 14.1}, \%\end{array}$ \\
\hline $9.4(400)$ & 25.2 & 22.2 & 54.4 \\
$11.7(500)$ & 32.2 & 28.8 & 75.6 \\
$14.1(600)$ & 35 & 31.6 & 100 \\
$16.4(700)$ & 38.8 & 35.3 & 125.4 \\
$21.9(930)$ & 46.6 & 43.1 & 193.6 \\
\hline
\end{tabular}

The relative sensitivities normalized with that for $14.1 \mathrm{~T}$,

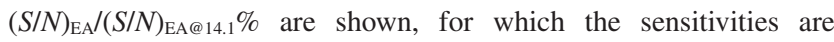
assumed to be proportional to $B_{0}^{3 / 2}$.

utilized for MQMAS experiments. Even in that case, adjustment of the spectral resolution using fullerene is favorably applicable. Although high-power ${ }^{1} \mathrm{H}$ decoupling can be applied during shimming for samples containing ${ }^{1} \mathrm{H}$ nuclei, long decoupling necessary to achieve high spectral resolution might damage to the probe and other equipment.

We dare to point out the unfavorable aspects of fullerene, such as its lower sensitivity at lower magnetic fields and a slightly high cost of 400 US dollars per $1 \mathrm{~g}$, although it is commercially available with ease.

\section{Conclusions}

In this article, it is demonstrated that fullerene is useful for the adjusting the spectral resolution for MQMAS experiments; ${ }^{1} \mathrm{H}$ decoupling is irrelevant to observe its ${ }^{13} \mathrm{C}$ signal, and so shimming can be performed using single-resonance MQMAS probes. There is no risk to damage probes and other equipment, caused by long high-power decoupling pulses. We can enjoy an acquisition time as long as necessary. The intrinsic ${ }^{13} \mathrm{C}$ MAS resonance line of fullerene is found to be sharp enough to adjust shimming. Indeed, the relaxation time $\left(T_{1}\right)$ of fullerene is long, 
but it becomes possible to shim at every $5 \mathrm{~s}$ with sufficiently high signal sensitivity if the appropriate Ernst angle is applied.

\section{References}

1. L. Frydman and J. S. Harwood, J. Am. Chem. Soc., 1995, 117, 5367.

2. A. Medek, J. S. Harwood, and L. Frydman, J. Am. Chem. Soc., 1995, 117, 12779.

3. J.-P. Amoureux, C. Fernandez, and L. Frydman, Chem. Phys. Lett., 1996, 259, 347.

4. J.-P. Amoureux, M. Pruski, D. P. Lang, and C. Fernandez, J. Magn. Reson., 1998, 131, 170 .
5. F. H. Larsen and N. C. Nielsen, J. Phys. Chem., 1999, 103, 10825.

6. R. Tycko, G. Dabbagh, R. Fleming, R. Haddon, A. Makhija, and S. Zahurak, Phys. Rev. Lett., 1991, 67, 1886.

7. K. Mizoguchi, J. Phys. Chem. Solids, 1993, 54, 1693.

8. V. P. Tarasov, Y. B. Muravlev, and D. E. Izotov, Phys. Rev. B, 2002, 66, 33407.

9. D. E. Izotov and V. P. Tarasov, J. Phys. Chem. B, 2002, 106, 5335.

10. R. R. Ernst, G. Bodenhausen, and A. Wokaun, "Principles of Nuclear Magnetic Resonance in One and Two Dimensions, Clarendon", 1988, Oxford University Press, New York, 125. 